\title{
A RESIDUAL PROPERTY OF CERTAIN LINEAR GROUPS
}

\author{
PETER F. STEBE
}

\begin{abstract}
An extension of residual finiteness, residual finiteness with respect to nests, is demonstrated for certain subgroups of $G L(n, Z)$, the polycyclic by finite groups. It is also shown that groups containing a free subgroup of rank greater than 1 cannot have the property. It is not settled whether or not there are other solvable by finite groups, subgroups allowed by Tits' theorem, that are residually finite with respect to nests.
\end{abstract}

Let $G$ be a group. If $N$ is a subset of $G \times G$ which contains $\left(a c^{-1}, d^{-1} b\right)$ whenever it contains $(a, b)$ and $(c, d)$, then $N$ is called a nest in $G \times G$. The set of all elements $a b$ in $G$ corresponding to the elements $(a, b)$ is called a nest in $G$ and the image of the nest in $G \times G$. An example of a nest is the set of all group elements which are equal to $x^{-1} \alpha(x)$ for a fixed automorphism $\alpha$ of $G$ and some element $x$ of $g$. The subset of $G \times G$ of all pairs $\left(x^{-1}, \alpha(x)\right)$ has the defining property of a nest, i.e. $\left(x^{-1}, \alpha(x)\right)$ and $\left(y^{-1}, \alpha(y)\right)$ combine to yield $\left(x^{-1} y, \alpha(y)^{-1} \alpha(x)\right)=\left(x^{-1} y, \alpha\left(y^{-1}, x\right)\right)$, which is again in the nest. If $\alpha$ is an inner automorphism of $G$, i.e. $\alpha(x)=g x g^{-1}$ for a fixed element $g$ of $G$ then $x^{-1} \alpha(x)=x^{-1} g x g^{-1}$, so that the corresponding nest is the set obtained by multiplying each element of the conjugacy class of $g$ in $G$ by $g^{-1}$. If we were to let $x$ represent only an element of a given subgroup $S$ of $G$, we would obtain the set of translates by $g^{-1}$ of the set of conjugates of $g$ by elements of $S$. If $S$ is a subgroup of $G$ then $S$ is obviously a nest in $G$. Clearly, the product complex of two subgroups $S_{1}$ and $S_{2}$ is a nest and the double coset $S_{1} g S_{2}$ is the translate of the product complex $S_{1} g S_{2} g^{-1}$. A less familiar example is provided by the set of all elements of $G$ which can be expressed as a palindrome of even length in terms of a subset of $G$ which contains the inverse of each of its elements.

The structure of nests in an arbitrary group was given by the author [15] as follows:

Let $S_{1}$ and $S_{2}$ be subgroups of a group $G$. Let $K_{i}$ be a normal subgroup of $S_{i}$ for $i=1,2$ such that $S_{1} / K_{1}$ is isomorphic to $S_{2} / K_{2}$. Let $\phi$ be an isomorphism of $S_{1} / K_{1}$ onto $S_{2} / K_{2}$. The set of all elements of $G \times G$ of the form $\left(a c, \phi\left(c^{-1}\right) b\right)$ with $a \in K_{1}, b \in K_{2}$ and $c$ a coset representative of $S_{1}$ modulo $K_{1}$ is a nest in $G \times G$. Conversely, every nest in $G \times G$ can be obtained by this construction. $S_{1}$ is called the left subgroup of the nest and $S_{2}$ the right subgroup.

A group $G$ is residually finite with respect to nests if for each nest $N$ in $G$ and each element of $g$ of $G$ not contained in $N$ there is a homomorphism $\phi$ of $G$ onto a finite group such that $\phi(g)$ is not contained in $\phi(N)$. In the topology of residually finite groups given by M. Hall [3], a set is closed if and only if for each element

Received by the editors February 5, 1986 and, in revised form, July 17, 1986.

1980 Mathematics Subject Classification (1985 Revision). Primary 20E26, 20F 10.

Key words and phrases. Residual property, linear group, decision problem. 
not in the set there is a homomorphism of the group onto a finite group such that the image of the element is not in the image of the set. It follows from the ideas of J. C. C. McKinsey and V. Dyson, cf. A. Mostowsky [10] and R. Lyndon and P. Schupp [9], that if a recursively enumerable set is closed in the M. Hall topology, then it is recursive, i.e. it can be decided whether or not a given element lies in the set. Since the set $\{1\}$ is a nest, groups which are residually finite with respect to nests are residually finite. The author has shown [15], that finitely generated nilpotent groups are residually finite with respect to nests. Not many broad classes of groups can be residually finite with respect to nests, as is shown by the following sequence of theorems.

THEOREM 1a. If a group is residually finite with respect to nests, all of its factor groups are residually finite.

This follows from the fact that every subgroup and hence every normal subgroup is a nest.

A nest in a group $G$ is generated by pairs $\left(u_{i}, v_{i}\right)$ of $G \times G$ if it is the smallest nest of $G \times G$ containing the pairs, i.e. the subset of $G \times G$ obtained by iterating the nesting operation $\nu$ : $(a, b) \nu(c, d)=\left(a c^{-1}, d^{-1} b\right)$ starting with the given pairs. Note that the nest contains $(1,1)=\left(u_{i} u_{i}^{-1}, v_{i}^{-1} v_{i}\right)=\left(u_{i}, v_{i}\right) \nu\left(u_{i}, v_{i}\right)$ and hence $\left(u_{i}^{-1}, v_{i}^{-1}\right)=(1,1) \nu\left(u_{i}, v_{i}\right)$. Thus in the notation of the structure theorem, the left subgroup $S_{1}$, is generated by the $u_{i}$ and the right subgroup $S_{2}$ is generated by the $v_{i}$. The nest is said to be finitely generated if it is generated by finitely many pairs.

If we restrict ourselves to finitely generated nests, we still have the following:

THEOREM 1b. If a finitely generated group $G$ is residually finite with respect to finitely generated nests, then every factor group of $G$ obtained by adding finitely many relations to the relations of $G$ is residually finite.

According to this result, if finitely generated free groups were residually finite with respect to nests then all finitely presented groups would be residually finite. Since finitely generated free groups are conjugacy separable, cf. [13], this shows that conjugacy separability does not imply residual finiteness with respect to nests. Theorem $1 \mathrm{~b}$ is equivalent to the following lemma.

LEMMA 1. The normal closure of a finite set of elements of a finitely generated group is a finitely generated nest.

Let the finitely generated group $G$ have generators $x_{i}, i=1, \ldots, n$, and let $R_{r}$, $r=1, \ldots, k$, be elements of the group. Let $N$ be generated by the pairs $\left(x_{i}, x_{i}^{-1}\right)$ for all $i$ and $\left(R_{r}, 1\right)$ for all $r$. If $g$ is a group element of length 1 or more and $g=$ $x_{i} h$ where $\varepsilon$ is +1 or -1 and $h$ has shorter length in terms of the generators than $g$, then

$$
\begin{aligned}
\left(g R_{r}^{\eta} g^{-1}, 1\right) & =\left(x_{i}^{\varepsilon} h R_{r}^{\eta} h^{-1} x_{i}^{-\varepsilon}, 1\right)=\left(x_{i}^{\varepsilon} h R_{r}^{\eta} h^{-1} x_{i}^{-\varepsilon}, x_{i}^{\varepsilon} x_{i}^{-\varepsilon}\right) \\
& =\left[\left(x_{i}^{\varepsilon}, x_{i}^{-\varepsilon}\right) \nu\left(h R_{r}^{-\eta} h^{-1}, 1\right)\right] \nu\left(x_{i}^{\varepsilon}, x_{i}^{-\varepsilon}\right) .
\end{aligned}
$$

Here $\varepsilon=1$ or $-1, \eta=1$ or -1 . Since $h$ has length shorter than that of $g$, it follows by induction that $g R^{\eta} g^{-1}$ and hence the normal closure of the $R_{r}$ is in $N$. Conversely, every element of $N$ is in the normal closure of the $R_{r}$. This follows by an easy induction on the number of $\nu$ operations used to obtain the element from the 
generators. Clearly if only one $\nu$ operation is used to obtain $(a, b), a b=1$ or $R_{r}$ for some $r$. If $a b$ is in the normal closure of the set $\left\{R_{r}\right\}$ and $a b$ is obtained from $(a, b)$ in the nest in $G \times G$ then $(a, b) \nu\left(x_{i}, x_{i}^{-1}\right)=\left(a x_{i}^{-1}, x_{i} b\right)$, which has image $a x_{i}^{-1} x_{i} b=a b$ in $G,(a, b) \nu\left(R_{r}, 1\right)=\left(a R_{r}^{-1}, 1 b\right)$ which has image $a R_{r}^{-1} b=a R_{r} a^{-1} a b$, which is in the normal closure of the set $\left\{R_{r}\right\}$.

THEOREM 2. If a group is residually finite with respect to nests, so is every subgroup.

This follows immediately from the fact that every nest in a subgroup of a group is also a nest in the group, and every homomorphism of a group can be restricted to a subgroup.

According to J. Tits [16], a finitely generated linear group either has a free subgroup of rank at least 2 and hence arbitrary finite rank or is solvable by finite. Since no free group of rank greater than 1 can be residually finite with respect to nests, it follows that if a finitely generated linear group is residually finite with respect to nests, it must be solvable by finite. We will see below that polycyclic by finite groups are residually finite with respect to nests.

THEOREM 3. If $H$ is a subgroup of finite index in a group $G$ and $H$ is residually finite with respect to nests, so is $G$.

According to a well-known result, $H$ contains a normal subgroup of finite index in $G$, the intersection of the finitely many conjugates of $H$, called the core of $H$. Since every subgroup of $H$ is again residually finite with respect to nests, we may assume that $H$ is normal in $G$.

Let $N$ be a nest in $G$. Let $N$ also denote the preimage of this nest in $G \times G$. The context will make clear which is meant in every phrase. Let $g$ be an element of $G$ not contained in $N$. We wish to find a normal subgroup $M$ of finite index in $G$ such that $g$ is not in $N M$. If $G$ is not in $N H$, we may take $M$ to be $H$. If $G$ is in $N H$, there is a pair $(a, b)$ of $N$ such that $g=a b h$ for some element $h$ of $H$. Hence $a^{-1} g b=b h b^{-1}$. If $a^{-1} g b^{-1} M$ meets $N M$ then $g b^{-1} M b=g M$ meets $a N M b=a N b M=N M$ and conversely, so $g$ may be replaced by $b h b^{-1}$. Since $H$ is normal, we may assume that $g$ is an element of $H$.

In the above paragraph we used the result that if $(a, b)$ is in a nest $N$ in $G \times G$, then the subsets $a N b$ and $N$ of $G$ are the same. If $(r, s)$ is in $N$, so is $\left(r^{-1}, s^{-1}\right)=$ $((r, s) \nu(r, s)) \nu(r, s)$ and $\left(a^{-1} r, s b^{-1}\right)=\left(a^{-1}, b^{-1}\right) \nu\left(r^{-1}, s^{-1}\right)$, so $a a^{-1} r s b^{-1} b=r s$ is in $a N b$. Thus $a N b$ contains $N$. Similarly, we can show that $N$ contains $a N b$.

Consider the intersection of the nest $N$ in $G$ with the subgroup $H$. It consists of the image of all pairs $(a, b)$ of $N$ in $G \times G$ such that $a b$ is an element of $H$. Select a coset representative system for $G$ modulo $H$ so that for each of the pairs $(a, b)$ of $N$ there is a pair $\left(c, c^{-1} h\right)$ of $N$ with $c$ a coset representative of $G$ modulo $H, h$ an element of $H$, such that $a \equiv c \bmod H$. If $a b$ is in $H$ it follows that $b \equiv c^{-1} \bmod H$. The intersection of $N$ with $H$ consists of finitely many translates of nests in $H$ (i.e. images of nests in $H \times H$ ) constructed as follows. If $c$ is a coset representative of $G$ modulo $H$, let $U_{c}$ be the set of all pairs $(x, y)$ in $N$ such that $x y$ is in $H$ and $x$ is congruent to $c$ modulo $H$. The intersection of $N$ and $H$ is the union of all the $U_{c}$. Note that the $U_{c}$ need not be nests. By the construction of the coset representative system, if $U_{c}$ is nonempty, there is a pair $\left(c, c^{-1} h\right)$ in $N$ with $h$ in $H$. The set 
$c^{-1} U_{c} h^{-1} c=T_{c}$ is a nest in $H \times H$, for if $\left(c^{-1} x_{1}, y_{1} h^{-1} c\right)$ and $\left(c^{-1} x_{2}, y_{2} h^{-1} c\right)$ are in $T_{c}$ then so is their $\nu$ product

$$
\left(c^{-1} x_{1} x_{2}^{-1} c, c^{-1} h y_{2}^{-1} y_{1} h^{-1} c\right)=\left(c^{-1}\left(x_{1} x_{2}^{-1} c\right),\left(c^{-1} h y_{2}^{-1} y_{1}\right) h^{-1} c\right) .
$$

Note that $x_{1} x_{2}^{-1} c \equiv c \bmod H$ and $c^{-1} h y_{2}^{-1} y_{1} \equiv c^{-1} \bmod H$. Since $g$ is not an element of $N, c^{-1} g h^{-1} c$ is not an element of $T_{c}$. Thus there is a normal subgroup of finite index in $H$, and hence a normal subgroup of finite index in $G$ (recall that $H$ is of finite index in $G$ ), call it $M_{c}$, such that $c^{-1} g h^{-1} c$ is not in $T_{c} M_{c}=c^{-1} U_{c} h^{-1} c M_{c}$, so $g$ is not in $U_{c} M_{c}$. Since there are but finitely many $M_{c}$, their intersection $M$ is also of finite index in $G$ and $g$ is not in $N M$. This completes the proof.

The $S$ groups of K. Hirsch $[\mathbf{5}, \mathbf{6}, \mathbf{7}]$, solvable groups that satisfy the maximal condition are sometimes called polycyclic. According to A. $\mathrm{Mal}^{\prime} \mathrm{cev}$ [8], each such group has a normal subgroup of finite index $G$ such that $G \backslash G^{\prime}$ is a finitely generated Abelian group and $G^{\prime}$ is nilpotent $\left(G^{\prime}\right.$ is the commutator subgroup of $G$ ). The following lemmas and the result of E. Formanek [2] and V. Remeslennikov [12] that polycyclic groups are conjugacy separable will be used to show that polycyclic groups are residually finite with respect to nests.

LEMMA 2. Let $G$ be a group, and let $Z\left(G^{\prime}\right)$ be the center of the commutator subgroup $G^{\prime}$ of $G$. Let $N$ be a nest in $G$. There is an isomorphism $\phi$ of $Z\left(G^{\prime}\right)$ onto the subgroup $K$ of a split extension $H$ of an Abelian group $I$ by an Abelian group $K$, and a nest $M$ of $H$ such that $I$ is the left subgroup of $M$ and $M=\phi\left(N \cap Z\left(G^{\prime}\right)\right)$. If the left subgroup of $N \cap Z\left(G^{\prime}\right)$ is finitely generated, then $I$ is finitely generated.

Note that it follows readily from the definition of a nest that the intersection of a nest in $G$ with any normal subgroup of $G$ is again a nest in $G$. Let $S$ be the normal subgroup, $N$ the nest and let $(a, b)$ and $(c, d)$ be elements of $N$ in $G \times G$ such that $a b$ and $c d$ are in $S$. The $\nu$ product $(a, b) \nu(c, d)=\left(a c^{-1}, d^{-1} b\right)$ has image $a c^{-1} d^{-1} b=a b \cdot b^{-1} c^{-1}\left(d^{-1} d^{-1}\right) c b$ which is in $S$ since $S$ is normal. Note that the left and right subgroups of $N$ need not lie in $S$, which complicates the proof of Theorem 3. In the present case $N \cap Z\left(G^{\prime}\right)$ is a nest in $G$.

The group $H$ will be constructed by giving its generators and relations. Let $z_{j}$ for $j$ in an index set generate $Z\left(G^{\prime}\right)$ and let $x_{i}$ for $i$ in an index set generate the left subgroup $S$ of $N \cap Z\left(G^{\prime}\right)$. Let the group $H$ be generated by new generators $z_{j}^{\prime}$ and $x_{i}^{\prime}$. Note that the mark' gives a 1-1 correspondence between the marked and unmarked generators. The relations of $H$ are

(1) the relations of $Z\left(G^{\prime}\right)$ with each generator replaced by the corresponding marked generator,

(2) the relations of $S / S \cap G^{\prime}$ with each generator replaced by the corresponding marked generator.

Since $Z\left(G^{\prime}\right)$ is a normal subgroup of $G$, there are elements $c_{i, j, \varepsilon}$ of $Z\left(G^{\prime}\right)$ equal to $x_{i}^{-\varepsilon} z_{j} x^{\varepsilon}$. Impose relations

(3) $x_{i}^{\prime-\varepsilon} z_{j}^{\prime} x_{i}^{\iota \varepsilon}=c_{i, j, \varepsilon}^{\prime}$ where $c_{i, j, \varepsilon}^{\prime}$ is obtained from $c_{i, j, \varepsilon}$ by replacing each occurrence of an unmarked generator by a marked generator.

If only relations (1) were imposed, the assignment $\phi\left(z_{j}\right)=z_{j}^{\prime}$ for all $j$ would define $\phi$ as an isomorphism of $Z\left(G^{\prime}\right)$ onto $K$, the kernel of $H$. Since $Z\left(G^{\prime}\right)$ is central in $G^{\prime} . G / G^{\prime}$ and hence $S G^{\prime} / G^{\prime}$ which is isomorphic to $S / S \cap G^{\prime}$ acts as a group of automorphisms of $Z\left(G^{\prime}\right)$. so that the imposition of the second and third 
sets of relations does not destroy the isomorphism between $Z\left(G^{\prime}\right)$ and $K$, for $H$ is a preimage of a semidirect product. Thus $\phi$ as defined above is an isomorphism of $Z\left(G^{\prime}\right)$ onto $K$. We extend $\phi$ to $S / S \cap G^{\prime}$ by setting $\phi\left(x_{j}\right)=x_{j}^{\prime}$. Thus $\phi$ restricts to an isomorphism of $S / S \cap G^{\prime}$ onto the subgroup $I$ of $H$. Note that $\phi$ is the marking operation.

Now we consider the image of the nest $N \cap Z\left(G^{\prime}\right)$ under the mapping $\phi$. Let $\theta$ be the restriction of the natural homomorphism of $G$ onto $G / G^{\prime}$ to $S$, followed by the marking operation $\phi$. Let $M$ be the nest in $H \times H$ consisting of all pairs $\left(\theta(a), \theta(a)^{-1} \phi(a b)\right)$ for $(a, b)$ in $N \cap Z\left(G^{\prime}\right) . \quad M$ is a nest in $H \times H$, for if $(c, d)$ and $(e, f)$ are pairs of $M$, there are pairs $(t, u)$ and $(v, w)$ of $N \cap Z\left(G^{\prime}\right)$ in $G \times G$ such that $c=\theta(t), d=\theta\left(t^{-1}\right) \phi(t u), e=\theta(v), f=\theta\left(v^{-1}\right) \phi(v w)$. The proof that $(c, d) \nu(e, f)=\left(c e^{-1}, f^{-1} d\right)$ is an element of $M$ results from the following:

$$
\begin{aligned}
\left(c e^{-1}, f^{-1} d\right) & =\left(\theta(t) \theta\left(v^{-1}\right), \phi(v w)^{-1} \theta(v) \theta(t)^{-1} \phi(t u)\right) \\
& =\left(\theta\left(t v^{-1}\right), \theta\left(t v^{-1}\right)^{-1} \phi\left(t v^{-1} w^{-1} u\right)\right),
\end{aligned}
$$

because of the identities

$$
\begin{aligned}
\theta\left(t v^{-1}\right)^{-1} \phi\left(t v^{-1} w^{-1} u\right) & =\theta\left(v t^{-1}\right) \phi\left(t v^{-1}\right) \phi\left(w^{-1} v^{-1}\right) \phi\left(v t^{-1}\right) \phi(t u) \\
& =\theta\left(v t^{-1}\right) \phi\left(v t^{-1}\right)^{-1} \phi\left(w^{-1} v^{-1}\right) \phi\left(v t^{-1}\right) \phi(t u) \\
& =\theta\left(v t^{-1}\right) \theta\left(v t^{-1}\right)^{-1} \phi\left(w^{-1} v^{-1}\right) \theta\left(v t^{-1}\right) \phi(t u) \\
& =\phi(v w)^{-1} \theta(v) \theta(t)^{-1} \phi(t u) .
\end{aligned}
$$

Note that if $g$ is an element of $Z\left(G^{\prime}\right)$ and $s$ is an element of $S$, then $\phi\left(s^{-1} g s\right)=$ $\theta(s)^{-1} \phi(g) \theta(s)$. Thus $M$ is a nest in $H$ and is $\phi\left(N \cap Z\left(G^{\prime}\right)\right)$.

In the proof of Lemma 3 below, the following result of the Higman-NeumannNeumann construction will be used. Let $G$ be a group and let $\alpha$ be an automorphism of $G$. Let $G$ be generated by $g_{j}$ for $j$ in an index set. Let a new group $G^{\prime}$ be generated its elements $c$ and $g_{j}^{\prime}$, with $j$ in the same index set as before. Let the relations of $G^{\prime}$ be the relations of $G$ with each $g_{j}$ replaced by the corresponding $g_{j}^{\prime}$, and the relations $c^{-1} g_{j}^{\prime} c=\alpha\left(g_{j}^{\prime}\right)$ where the word on the right is obtained from $\alpha\left(g_{j}\right)$ by replacing each $g$ by the corresponding $g^{\prime}$. The replacement of each $g$ by the corresponding $g^{\prime}$ defines an isomorphism of $G$ onto the subgroup of $G^{\prime}$ generated by the $g^{\prime}$. See B. H. Neumann [11].

LEMMA 3. Let $H$ be a split extension of an Abelian group I by an Abelian group $K$. Let $N$ be a nest contained in $K$, such that the left subgroup of $N$ is $I$. The right kernel $K_{2}$ is a normal subgroup of $H$ and $N K_{1}=N$. Let $\gamma$ be the natural homomorphism of $H$ onto $H^{*}=H / K_{2}$. There is an automorphism $\psi$ of $H^{*}$ such that $\gamma(N)$ is the set of all products $x \psi\left(x^{-1}\right)$ for $x$ ranging over $H^{*}$. There is an embedding $\delta$ of $H^{*}$ into a cyclic extension $H^{* *}=\left\langle c, H^{*}\right\rangle$ of $H^{*}$ such that $H^{* *}$ is a split extension of an Abelian group by an Abelian group and $\delta \gamma(N)$ is the set of all $h c^{-1} h^{-1} c$ for $h$ ranging over $H^{* *}$.

PROOF. First, $K_{1}$ is a normal subgroup of $H$. Let $h$ and $k$ be elements of $H$ and $K_{1}$ respectively. There are elements $x$ of $I$ and $y$ of $K$ such that $h=y x$. Now $h^{-1} k h=x^{-1} y^{-1} k y x=x^{-1} k x$, since $K$ is Abelian and $K_{1} \subseteq N \cap K$. Since $I$ is the left subgroup of $N$, there is a pair $(x, u)$ in $N$. Since $x u=k_{1} \in K, u=$ $x^{-1} k_{1}$ and $u k u^{-1}=x^{-1} k_{1} k k_{1}^{-1} x=x^{-1} k x$, since $K$ is Abelian. Since $(1, k) \in N$, 
$\left(1, u k u^{-1}\right)=\left(x^{-1} 1 x, u k u^{-1}\right)$ is a pair of $N$, and $h^{-1} k h=x^{-1} k x=u k u^{-1} \in K_{1}$. Also $N K_{2}=N$. Let $(a, b)$ be a pair in $N$. Let $k$ be in $K_{2}$. Thus $(1, k)$ is in $N$, and so $(a 1, b k)$ is in $N$, so that $a b k$ is in $N$ (in $H$ ).

Let $\gamma$ and $H^{*}$ be defined as in the statement of the lemma. The image $\gamma(N)$ has trivial left and right kernels. First, $\gamma(N)$ is the set of all pairs $(\gamma(a), \gamma(b))$ for $(a, b)$ in $N$. Since the kernel of $\gamma$ is $K_{2}, K, \gamma$ is $1-1$ on $I$ and $\gamma(I)=I^{*}$ is an Abelian subgroup of $H^{*}$. Since $N \subset K, \gamma(N) \subset \gamma(K)=K^{*}$, and $H^{*}$ is the split extension of $I^{*}$ by $K^{*}$. The right kernel of $\gamma(N)$ is the identity. Let $(1, u)$ be a pair in $\gamma(N)$. There is a pair $(a, b)$ in $N$ such that $1=\gamma(a), u=\gamma(b)$. Since $\gamma$ is $1-1$ on $I, a=1$, so $b \in K_{1}$. Since $K_{1}$ is the kernel of $\gamma, u=1$. The left kernel of $\gamma(N)$ is also the identity, for if $(u, 1)$ is in $\gamma(N)$, then $u$ is in $\gamma(I)$, and $u=u 1$ is in $\gamma(K)$. But $\gamma(K)$ and $\gamma(I)$ meet only in the identity, for if $u=\gamma(i)=\gamma(k), k \in K, i \in I$, then $\gamma\left(k i^{-1}\right)=1$, so $k i^{-1} \in K_{2}$ and so $i \in K$. Thus $i=1$, so $u=1$.

Let $\phi$ be the isomorphism of the nest $\gamma(N)$, as described in the structure theorem. Since both the left and right kernels of $\gamma(N)$ are the identity, $\gamma(N)$ consists of all pairs $\left(x, \phi(x)^{-1}\right)$, where $x$ ranges over the left subgroup of $\gamma(N)$. For $x \in I^{*}$, $x \phi\left(x^{-1}\right) \in \gamma(N) \subset K^{*}$, so $x \phi(x) \bmod K^{*}$. Every element $h$ of $H^{*}$ has a unique expression $h=x y$ with $x \in I^{*}, y \in K^{*}$. If $x_{1} y_{1}=x_{2} y_{2}$ are two such products, then $x_{2}^{-1} x_{1}=y_{2} y_{1}^{-1}$. Since the homomorphism of $H^{*}$ onto $I^{*}$ has kernel $K^{*}$ and fixes $I^{*}$, we must have $x_{2}^{-1} x_{1}=1$ and so $y_{2} y_{1}^{-1}=1$.

Define $\psi(h)=\phi(x) y$. We will see that $\psi$ is an automorphism of $H^{*}$. First, $\psi$ is onto, for let $h \in H^{*}, h=x y$ with $x \in I^{*}, y \in K^{*}$. Since $x \equiv \phi(x) \bmod K^{*}$, there is an element $u$ of $K^{*}$ such that $x=\phi(x) u$. Thus $h=\phi(x) u y=\psi(x u y)$. Next, $\psi$ is $1-1$, for let $h_{i}=x_{i} y_{i}, x_{i} \in I^{*}, y_{i} \in K^{*}$. If $\psi\left(h_{1}\right)=\psi\left(h_{2}\right)$ then $\phi\left(x_{1}\right) y_{1}=$ $\phi\left(x_{2}\right) y_{2}$, so $\phi\left(x_{2}\right)^{-1} \phi\left(x_{1}\right)=y_{2} y_{1}^{-1} \in K^{*}$, so $\phi\left(x_{2}\right)^{-1} \phi\left(x_{1}\right)=y_{2} y_{1}^{-1} \in K^{*}$. But $\phi\left(x_{2}\right)^{-1} \phi\left(x_{1}\right)=\phi\left(x_{2}^{-1} x_{1}\right) \equiv x_{2}^{-1} x_{1} \bmod K^{*}$, so $x_{2}^{-1} x_{1} \in K^{*}$. But $I^{*} \cap K^{*}$ is the identity so $x_{1}=x_{2}, y_{1}=y_{2}$ and therefore $h_{1}=h_{2}$. Finally, $\psi$ is a homomorphism, for let $h_{i}, x_{i}$ and $y_{i}$ be as above. Then $\psi\left(h_{1}\right) \psi\left(h_{2}\right)=\phi\left(x_{1}\right) y_{1} \phi\left(x_{2}\right) y_{2}$. Since $\phi\left(x_{2}\right) \equiv x_{2} \bmod K^{*}, \phi\left(x_{2}\right)^{-1} y_{1} \phi\left(x_{2}\right)=x_{2}^{-1} y_{1} x_{2}$ follows from the fact that $K^{*}$ is Abelian. Thus

$$
\begin{aligned}
\psi\left(h_{1}\right) \psi\left(h_{2}\right) & =\phi\left(x_{1}\right) \phi\left(x_{2}\right) x_{2}^{-1} y_{1} x_{2} y_{2}=\phi\left(x_{1} x_{2}\right) x_{2}^{-1} y_{1} x_{2} y_{2} \\
& =\psi\left(x_{1} x_{2} x_{2}^{-1} y_{1} x_{2} y_{2}\right)=\psi\left(h_{1} h_{2}\right) .
\end{aligned}
$$

Now $\gamma(N)$ is the set of all elements $h \psi(h)^{-1}$ for $h$ ranging over $H^{*}$. If $h \in H^{*}$, $h=x y$ with $x \in I^{*}, y \in K^{*}$, then

$$
h \psi(h)^{-1}=x y(\phi(x) y)^{-1}=x y y^{-1} \phi(x)^{-1}=x \phi(x)^{-1} .
$$

Add a new generator $c$ to the generators of $H^{*}$. For each generator $g$ of $H^{*}$, add the relation $c^{-1} g c=\psi(g)$. Since $\psi$ is an automorphism of $H^{*}$, the naturally defined homomorphism $\delta$ of $H^{*}$ into the new group $H^{* *}$ is an isomorphism. Since $\delta\left(H^{*}\right)$ is a normal subgroup of $H^{* *}$ such that $H^{* *} / \delta\left(H^{*}\right)$ is cyclic, $H^{* *}$ is a cyclic extension of $H^{*}$. The nest $\gamma \delta(N)$ is the set of all elements of the form $h c^{-1} h^{-1} c$ for $h$ ranging over $\delta\left(H^{*}\right)$, for we have seen above that $\gamma(N)$ is the set of all elements $h \psi(h)^{-1}$ so that $\delta \gamma(N)$ is the set of all $h c^{-1} h^{-1} c$. Clearly, $\delta \gamma(N) \subseteq \delta\left(K^{*}\right)$. Finally, $H^{* *}$ is a split extension of an Abelian group by an Abelian group. Let $A$ be the subgroup of $H^{* *}$ generated by $c$ and $\delta\left(K^{*}\right)$. Since $\psi(g)=g$ for every element $g$ of $K^{*}, c$ commutes with every element of $\delta\left(K^{*}\right)$, so $A$ is Abelian. $A$ is normal in $H^{* *}$, for 
$\delta\left(K^{*}\right)$ is normal in $H^{* *}$, and for all $h$ in $H^{* *}, h c^{-1} h^{-1}$ is in $\delta\left(K^{*}\right)$, so $h c^{-1} h^{-1}$ is an element of $A$. $H^{* *} / A$ is clearly $\delta\left(I^{*}\right)$, and hence is Abelian. This completes the proof of Lemma 3.

LEMMA 4. Let $G$ be a group. Let $Z\left(G^{\prime}\right)$ be the center of the commutator subgroup $G^{\prime}$ of $G$. Let $N$ be a nest in $G$ and let $g \in Z\left(G^{\prime}\right)$, but $g \notin N \cap Z\left(G^{\prime}\right)$. If $Z\left(G^{\prime}\right)$ and the left subgroup of $N \cap Z\left(G^{\prime}\right)$ are finitely generated, there is a subgroup of finite index in $Z\left(G^{\prime}\right)$ such that $g \notin\left(N \cap Z\left(G^{\prime}\right)\right) M$.

PROOF. Since the kernel of the composite of the embeddings of Lemmas 1 and 2 is such that the product of $N \cap Z\left(G^{\prime}\right)$ with the kernel is again $N \cap Z\left(G^{\prime}\right)$, we may assume that $G$ is a split extension of a finitely generated Abelian group by a finitely generated Abelian group and $N \cap Z\left(G^{\prime}\right)$ is the set of all elements $x c^{-1} x^{-1} c$ for $x$ in $G$. But $g=x c^{-1} x^{-1} c$ is equivalent to $g c^{-1}=x c^{-1} x^{-1}$. According to E. Formanek [1] and V. Remeslennikov [12], all polycyclic groups and hence all groups of this type are conjugacy separable, so that there is a subgroup of finite index in $G$ and hence there is a subgroup $M$ of finite index in $Z\left(G^{\prime}\right)$ such that $g c^{-1}$ is not equal to the product of a conjugate of $c^{-1}$ and an element of $M$. But then $g$ is not an element of $\left(N \cap Z\left(G^{\prime}\right)\right) M$.

LEMMA 5. Let $G$ be a finitely generated group. If the commutator subgroup $G^{\prime}$ of $G$ is finite, then the center $Z(G)$ of $G$ is of finite index in $G$.

Lemma 5 is well known, a special case of a theorem of P. Hall [4].

THEOREM 4. Every polycyclic group is residually finite with respect to nests.

PROOF. If a group is finitely generated and has a finitely generated nilpotent commutator subgroup, it is called an $N A$ group. By Theorem 3 and the result of $\mathrm{Mal}^{\prime} \mathrm{cev}$ cited above we can restrict our attention to $N A$ groups. Let $G$ be an $N A$ group. The proof is by induction on the Hirsch rank of $G^{\prime}$. If $G^{\prime}$ has Hirsch rank zero, it is finite, and it follows from Lemma 5 that the center of $G$ is of finite index in $G$. The center of $G$ is Abelian and finitely generated since it is a subgroup of a polycyclic group. Finitely generated Abelian groups are residually finite with respect to nests because their nests are subgroups and the groups are subgroup separable. Let $G^{\prime}$ have Hirsch rank greater than zero, and assume that every $N A$ group with commutator subgroup of lesser Hirsch rank is residually finite with respect to nests. Let $N$ be a nest in $G$ and let $g$ be an element of $G$ not contained in $N$. Suppose first that $g \notin N Z\left(G^{\prime}\right)$. According to P. Hall (cf. G. Baumslag [1, p. 6]), $Z\left(G^{\prime}\right)$ contains an element of infinite order. Thus the commutator subgroup of $G / Z\left(G^{\prime}\right)$ has lesser Hirsch rank than that of $G^{\prime}$, so by the inductive hypothesis, $G / Z\left(G^{\prime}\right)$ is residually finite with respect to nests. Thus there is a normal subgroup $M$ of finite index in $G / Z\left(G^{\prime}\right)$ such that $g \notin N Z\left(G^{\prime}\right) M Z\left(G^{\prime}\right)$. But $M Z\left(G^{\prime}\right)$ is of finite index in $G$. Now assume that $g$ is in $N Z\left(G^{\prime}\right)$. There is a pair $(a, b)$ of $N$ in $G \times G$ and an element $g_{1}$ of $Z\left(G^{\prime}\right)$ such that $g=a b g_{1}$, so $a^{-1} g b^{-1}=b g_{1} b^{-1}$ is in $Z\left(G^{\prime}\right)$. If $a^{-1} g b^{-1}=c d$ where $(c, d)$ is in $N$, then $g=a c d b$, an element of $N$. Hence $a^{-1} g b^{-1}$ is an element of $Z\left(G^{\prime}\right)$ not contained in $N$. According to Lemma 4 , there is a subgroup $M$ of finite index in $Z\left(G^{\prime}\right)$ such that $a^{-1} g b^{-1} \notin\left(N \cap Z\left(G^{\prime}\right)\right) M$. According to a theorem of M. Hall [3], there are only finitely many subgroups of a given index in a finitely generated group. Thus $M^{*}$, the intersection of all the subgroups of the same index as $M$ in $Z\left(G^{\prime}\right)$ is of finite index in $Z\left(G^{\prime}\right)$ and normal 
in $G$. Since $M \supset M^{*}, a^{-1} g b^{-1} \notin\left(N \cap Z\left(G^{\prime}\right)\right) M^{*}$. Then $a^{-1} g b^{-1} \notin N M^{*}$, for both $a^{-1} g b^{-1}$ and $M^{*}$ are contained in $Z\left(G^{\prime}\right)$. Since $M^{*}$ is a normal subgroup of $G, g \notin a N b M^{*}=N M^{*}$, where we use the fact that $(a, b)$ is in $N$. Again using the result of $\mathrm{P}$. Hall cited above, the Hirsch rank of the commutator subgroup of $G / M^{*}$ is less than that of $G^{\prime}$, so that as before there is a normal subgroup $S$ of finite index in $G$ such that $g \notin N S$.

THEOREM 5. Finite extensions of polycyclic groups are residually finite with respect to nests.

PROOF. This is obtained by using Theorem 2 in conjunction with Theorem 4 .

\section{REFERENCES}

1. G. Baumslag, Lecture notes on nilpotent groups, CBMS Regional Conf. Ser. in Math., no. 2, Amer. Math. Soc., Providence, R.I., 1971, pp. 1-73.

2. E. Formanek, Conjugate separability in polycyclic groups, J. Algebra 42 (1976), 1-10.

3. M. Hall, Jr., A topology for free groups and related groups, Ann. of Math. (2) 52 (1950), 127-139.

4. P. Hall, Finiteness conditions for soluble groups, Proc. London Math. Soc. 4 (1954), 419-436.

5. K. Hirsch, On infinite soluble groups. I, Proc. London Math. Soc. 44 (1938), 53-60.

6. __ On infinite soluble groups. II, Proc. London Math. Soc. 44 (1938), 336-344.

7. __ On infinite soluble groups. II, Proc. London Math. Soc. 49 (1946), 184-194.

8. A. I. Mal'cev, On certain classes of infinite soluble groups, Mat. Sb. 28 (1951), 567-588; English transl., Amer. Math. Soc. Transl. (2) 2 (1956), 1-21.

9. R. Lyndon and P. Schupp, Combinatorial group theory, Ergeb. Math. Grenzgeb., Band 98, Springer-Verlag, Berlin and New York, 1977.

10. A. W. Mostowski, On the decidability of some problems in certain classes of groups, Fund. Math. 59 (1966), 123-135.

11. B. H. Neumann, An essay on free products of groups with amalgamation, Philos. Trans. Roy. Soc. London Ser. A 246 (1954), 503-554.

12. V. N. Remeslennikov, Conjugacy in polycyclic groups, Algebra i Logica 8 (1969), 712-725.

13. P. F. Stebe, A residual property of certain groups, Proc. Amer. Math. Soc. 26 (1970), 37-42.

14. __ Residual solvability of an equation in nilpotent groups, Proc. Amer. Math. Soc. 54 (1976), $57-58$.

15.

16. J. Tits, Free subgroups in linear groups, J. Algebra 20 (1972), 250-270.

Department of Mathematics, City College (CUNy), New York, New York 10031 